Revue internationale P.M.E.

Économie et gestion de la petite et moyenne entreprise

\title{
L'engagement des entrepreneurs : des doutes au second souffle
}

\section{Patrick Valéau}

Volume 20, numéro 1, 2007

URI : https://id.erudit.org/iderudit/1008512ar

DOI : https://doi.org/10.7202/1008512ar

Aller au sommaire du numéro

Éditeur(s)

Presses de l’Université du Québec

ISSN

0776-5436 (imprimé)

1918-9699 (numérique)

Découvrir la revue

Citer cet article

Valéau, P. (2007). L’engagement des entrepreneurs : des doutes au second souffle. Revue internationale P.M.E., 20(1), 121-154.

https://doi.org/10.7202/1008512ar

\section{Résumé de l'article}

Devant les difficultés, les entrepreneurs se doivent de toujours persévérer, mais en pratique, la plupart d'entre eux passent par des périodes de doute au cours desquelles leur engagement se fait plus incertain. Paradoxalement, ces remises en question peuvent contribuer à la perpétuation de l'activité entrepreneuriale : s'arrêter et prendre le temps de douter peut permettre aux entrepreneurs de faire le point sur leurs attentes et de redécouvrir l'entreprise sous un nouveau jour. C'est très souvent à cette condition que les entrepreneurs reconstruisent l'engagement nécessaire pour poursuivre l'entreprise : un engagement souvent très différent de celui qui avait motivé sa création. À partir de 50 entretiens, cet article propose un modèle en quatre étapes : engagement anticipé, engagement dans l'action, engagement confus et engagement du second souffle. Partant de là, nous invitons les accompagnateurs et les partenaires du projet à soutenir les entrepreneurs dans ces moments difficiles afin de leur laisser le temps de retrouver la force de continuer.
Ce document est protégé par la loi sur le droit d'auteur. L'utilisation des services d’Érudit (y compris la reproduction) est assujettie à sa politique d'utilisation que vous pouvez consulter en ligne.

https://apropos.erudit.org/fr/usagers/politique-dutilisation/ 


\title{
L'engagement des entrepreneurs: des doutes au second souffle
}

\author{
Patrick VALÉAU \\ Université de La Réunion
}

\author{
MOTS CLÉS
}

\section{Engagement - Implication - Motivations - Doutes \\ Confusion - Abandon - Changement}

\begin{abstract}
L'AUTEUR
PatRICK ValÉau est enseignant-chercheur à l'Université de La Réunion et directeur de la licence professionnelle d'entrepreneuriat dans les organisations à but non lucratif. Ses recherches portent sur l'engagement, l'accompagnement des entrepreneurs, les organisations à but non lucratif et la responsabilité sociale. Adresse: 9, rue des Pétrels, 97417 La Montagne, La Réunion, France. Courriel: <patrick@valeau.com>.
\end{abstract}

\section{RÉSUMÉ}

Devant les difficultés, les entrepreneurs se doivent de toujours persévérer, mais en pratique, la plupart d'entre eux passent par des périodes de doute au cours desquelles leur engagement se fait plus incertain. Paradoxalement, ces remises en question peuvent contribuer à la perpétuation de l'activité entrepreneuriale: s'arrêter et prendre le temps de douter peut permettre aux entrepreneurs de faire le point sur leurs attentes et de redécouvrir l'entreprise sous un nouveau jour. C'est très souvent à cette condition que les entrepreneurs reconstruisent l'engagement nécessaire pour poursuivre l'entreprise: un engagement souvent très différent de celui qui avait motivé sa création. À partir de 50 entretiens, cet article propose un modèle en quatre étapes: engagement anticipé, engagement dans l'action, engagement confus et engagement du second souffle. Partant de là, nous invitons les accompagnateurs et les partenaires du projet à soutenir les entrepreneurs dans ces moments difficiles afin de leur laisser le temps de retrouver la force de continuer.

\section{ABSTRACT}

Facing difficulties, entrepreneurs must always persevere but in practice, most of them go through periods of doubt during which their commitment becomes more uncertain. Paradoxically, these doubts can help the perpetuation of the entrepreneurial activity: 
to stop and take time to doubt allows them to update their expectation and to see their venture in a new light. It is often through this step that entrepreneurs rebuild the commitment needed to carry on, this commitment being often different from the one that initially motivated them to start their business. Based upon 50 interviews, this article proposes a model in four stages: anticipated commitment, commitment to action, confused commitment and second wind commitment. Considering this process, partners and councillors are invited to support entrepreneurs when facing these hard times and to give them time to find the strength to carry on.

\section{RESUMEN}

Frente a las dificultades, los entrepreneurs se deben de perseverar siempre, pero en práctica, la inmensa mayoría de ellos pasan por períodos de duda en el curso de los cuales su empeño se hace más incierto. Paradójicamente, estas dudas pueden contribuir a la perpetuación de la actividad entrepreneuriale: pararse y tomarse un tiempo para dudar puede permitir a los entrepreneurs de redescubrir su empresa bajo un nuevo día. Es muy a menudo en esta condición que los entrepreneurs reconstruyen el empeño necesario para poder perseguir la empresa: un empeño a menudo muy diferente del que había motivado la creación. Teniendo como base 50 entrepreneurs, este artículo propone un modelo en cuatro etapas : empeño anticipado, empeño en la acción, el empeño confuso y el empeño del segundo soplo. Por lo tanto de ahí, invitamos a los acompañantes y los compañeros del proyecto que sostiene a los entrepreneurs en estos momentos difíciles con el fin de dejarles el tiempo de reencontrar la fuerza de continuar.

\section{ZUSAMMENFASSUNG}

Unternehmer müssen sich auch bei Schwierigkeiten behaupten können und die Realität zeigt, dass viele Unternehmer Phasen des Zweifels durchlaufen und ihr Engagement ernsthaft in Frage stellen. Paradoxerweise führt dieses In-Frage-Stellen zu einer Fortführung der unternehmerischen Aktivität: Inne zu halten und sich Zeit zu nehmen, erlaubt dem Unternehmer seine Erwartungen zu überdenken und sein Unternehmen in anderem Lichte zu betrachten. Vielfach führt diese Situation dazu, dass der Unternehmer sein Engagement neu definiert und sein Unternehmen anders weiterführt. Dieser Einsatz unterscheidet sich nicht selten grundsätzlich von der ursprünglichen Motivation der Unternehmungsgründung. Basierend auf 50 Gesprächen mit Unternehmern schlägt der Artikel ein Vierphasen-Modell zur Beurteilung des unternehmerischen Engagements vor: Vorausschauendes Engagement, Handlungseinsatz, unklares Engagement und Engagements des zweiten Atems. Von diesem Modell ausgehend, fordern wir Begleiter und Partner eines Projektes dazu auf, den Unternehmer in solchen kritischen Phasen zu unterstützen, damit er die nötige Kraft zur Fortsetzung findet. 


\section{Introduction}

L'entrepreneur est un individu qui innove, prend des risques et saisit des occasions d'affaires en créant son entreprise puis en maintenant son développement. Il est souvent envisagé comme un être à part: un individu farouchement indépendant, déterminé et tenace, trouvant la force de maintenir son effort envers et contre tous ${ }^{1}$. Les recherches en entrepreneuriat se sont naturellement intéressées, à travers différents concepts, à l'engagement de ces individus. Devant les difficultés d'établir un portrait universel et définitif de l'entrepreneur (Shaver, 1995), les chercheurs ont peu à peu conclu que tous les entrepreneurs ne créaient pas leur entreprise avec le même engagement. Au-delà de ces contingences, la plupart des textes évoquent des niveaux d'engagement particulièrement intenses, mais surtout la persévérance face aux difficultés de l'entreprise et la capacité à se remotiver et à redoubler d'efforts en cas d'échec.

Cet article reprend ces contributions en approfondissant un aspect peu valorisé et peu étudié de l'expérience entrepreneuriale: le doute, autrement dit la confusion et les baisses d'engagement. Trois questions sont ainsi abordées: Dans quelle mesure les entrepreneurs connaissent-ils le doute, la confusion et les baisses d'engagement? Comment les surmontent-ils? Dans quelle mesure ces phases de doute affectent-elles l'entreprise? Les «doutes » en question sont différents des «stress» ressentis face aux incertitudes et aux difficultés de l'entreprise (Boyd et Gumpert, 1983; Adebowale, 1994; Afzalur, 1996): le stress est une tension, une réaction physique et psychique motivante destinée à faire face au danger, alors que le doute correspond à une remise en cause plus «existentielle», à une perte de sens se traduisant par une baisse d'engagement.

Suivant les méthodologies qualitatives de l'entretien, nous avons repris les parcours de 50 entrepreneurs, tous encore relativement actifs. Au-delà de leurs différences, ces entrepreneurs se ressemblent dans les étapes du processus d'engagement suivi: presque tous passent par des phases d'engagement avec des motivations plus faibles, puis presque tous évoluent et reconstruisent l'engagement à poursuivre l'activité entrepreneuriale; presque tous changent. Dans une première partie, nous introduisons le concept d'engagement au sein de la littérature en entrepreneuriat. Dans la deuxième partie, nous présentons les méthodes qualitatives utilisées pour recueillir les données. Dans la troisième partie, nous confrontons nos données de terrain à la théorie, étape par étape, introduisant ainsi un modèle dialectique en quatre étapes entre persévérance et changement.

1. Nous reformulons ainsi le mythe de l'entrepreneur évoqué par Shaver (1995). 
La phase de doutes constitue un moment crucial de l'aventure entrepreneuriale. Au-delà de la santé économique des entreprises, elle touche le développement psychologique et social de ceux qui les impulsent. Durant cette période, les entreprises courent un double risque: tout d'abord les hésitations et la confusion qui caractérisent le doute apparaissent peu compatibles avec la détermination et la clairvoyance exigées par l'activité entrepreneuriale; il reste, ensuite, la possibilité que les entrepreneurs décident d'y mettre définitivement fin. Ainsi, chaque année, des entreprises déclarent forfait, à cause d'un découragement prématuré, alors qu'elles auraient sans doute pu survivre.

\section{L'engagement des entrepreneurs}

L'entrepreneuriat comprend au moins deux catégories de recherches: les premières se focalisent sur les activités liées à la création et au développement d'entreprises; les autres s'efforcent de mieux comprendre les individus à l'origine de ces comportements. Face à cette «dialogie» (Bruyat, 1994; Verstraete 2002), nous «importons» le concept d'engagement ${ }^{2}$ : un concept habituellement réservé à la gestion des ressources humaines. Nous réinterprétons en ces termes un certain nombre de contributions relatives aux expériences vécues par les entrepreneurs.

\subsection{Des activités entrepreneuriales à l'entrepreneur}

Une grande partie des recherches en entrepreneuriat étudient ce que «fait» effectivement l'entrepreneur (Gartner, 1998). Dans ce sens, l'entrepreneuriat peut être défini comme un ensemble d'activités associées à la création et au développement d'une entreprise. Ainsi, l'entrepreneur est avant tout un innovateur (Schumpeter, 1935) et un preneur de risques (Mill, 1848; Say, 1803; Knight, 1921) sans cesse à la recherche de nouvelles occasions d'affaires à exploiter (Cantillon, 1755; Say, 1803 ; Kirzner, 1993). Ces approches produites par les auteurs classiques des siècles passés forment encore aujourd'hui le cœur de la littérature sur l'entrepreneuriat. Pour de nombreux auteurs, ces activités caractérisent la phase de création (Van de Ven, Huston et Schroeder, 1984; Bygrave et Hofer, 1991; Gartner, Bird et Starr, 1992; Gartner et Gatewood, 1992; Hofer et Bygrave, 1992; Davidson, Low et Wright, 2001). Pour d'autres la phase entrepreneuriale se poursuit aussi longtemps que l'entrepreneur saisit de nouvelles opportunités (Filion, 1997 ; Shane et Vankatamaran, 1997; Ucbasaran, Westhead et Wright, 2001) et développe

2. Dans son ouvrage D'une science à l'autre, des concepts nomades, Stengers (1987) montre la fertilité de telles importations.

Revue internationale P.M.E., vol. 20, nº 1, 2007 
son entreprise (Carland et al., 1984; Shane et Vankatamaran,1997; Davidson et Wiklund, 2001). Pour ces auteurs, le défi de l'entrepreneuriat consiste à perpétuer l'entreprise au moins autant qu'à la créer (Naffziger, Hornsby et Kurato, 1997). Dans ce sens, la phase entrepreneuriale se poursuit aussi longtemps que l'individu «impulse » l'entreprise (Verstraete, 2002).

Partant de là, de nombreuses contributions se sont intéressées aux qualités communes aux individus à l'origine de ces activités. Brockhaus et Horwitz (1985) résument ces recherches empiriques: l'entrepreneur est un individu caractérisé par le besoin de réalisation de soi; l'indépendance et un locus de contrôle interne sont des éléments effectivement présents chez beaucoup d'entrepreneurs. Ces recherches se poursuivent encore aujourd'hui: certaines confirment la spécificité de la personnalité des entrepreneurs (p. ex., Johnson, 1990); d'autres montrent que ces derniers ne sont pas forcément très différents des non-entrepreneurs (Vesalainen et Pihkala,1999; Gartner, Shaver et Gatewood,2000). Des auteurs comme Shaver (1995) remettent, ce faisant, en cause certains mythes concernant les entrepreneurs. Face aux difficultés d'aller plus loin dans la mise au jour de ces caractéristiques communes et distinctives des entrepreneurs, de plus en plus de textes mettent en avant la diversité des entrepreneurs: pour Gartner et al. (1994), par exemple, l'entrepreneur est probablement pluriel, il y a de nombreux types différents d'entrepreneurs, il n'y a pas de moyenne dans l'entrepreneuriat, l'entrepreneuriat est idiosyncratique. En fait, les typologies contribuaient depuis déjà longtemps à une construction de la connaissance des phénomènes entrepreneuriaux allant dans le sens de la diversité. Depuis, la distinction établie par Smith (1967) entre «entrepreneur artisan » et «entrepreneur opportuniste » et les 11 styles de Vesper (1980), ces typologies se sont progressivement imposées (Filion, 1997, 2000a, 2000b), affirmant certaines pluralités des phénomènes entrepreneuriaux.

Cette ouverture sur la diversité des entrepreneurs ne va cependant pas sans critiques, les «puristes» lui reprochent de «galvauder» le concept en intégrant toutes sortes de dirigeants-propriétaires, sans tenir compte du développement et des performances actuelles de l'entreprise (Carland et al., 1984; Van de Ven, Huston et Schroeder, 1984; Gartner, 1998). De leur côté, les partisans de l'ouverture reprochent aux puristes leurs approches réductrices perdant de vue l'individu-entrepreneur. On assiste depuis le début des années 1990 à l'institution d'un clivage entre deux corpus se considérant incompatibles.

Devant cette incommensurabilité, certains chercheurs francophones suivent une réflexion ontologique et épistémologique différente: ils développent des modèles de l'entrepreneuriat fondés sur une distinction entre 
l'entrepreneur et l'entreprise, leurs logiques peuvent être différentes mais n'en restent pas moins interdépendantes. Sur le plan conceptuel, Verstraete (2002) définit l'entrepreneuriat comme une dialectique cognitive, praxéologique et structurale mettant en relation l'entrepreneur et son entreprise. Sur un plan plus pratique, Bruyat (1994) met en perspective les interactions dialogiques entre la condition de l'entrepreneur et les innovations liées à son projet d'entreprendre. Dans ce sens, l'entrepreneur «impulse» l'organisation, il la conçoit tout d'abord sous la forme d'une vision qu'il réalise ensuite (Filion, 1997). Mais il ne se réduit pas à ce comportement: au-delà de l'activité entrepreneuriale, il reste un individu à part entière. Ces cadres d'analyse permettent de penser simultanément la diversité de l'entrepreneur et des activités entrepreneuriales contribuant au développement de l'entreprise. Nous proposons, à présent, de poursuivre dans ce sens en important le concept d'engagement tel qu'il a été développé en gestion des ressources humaines.

\subsection{Le concept d'engagement ${ }^{3}$}

L'engagement est un concept qui décrit la relation entre l'individu et son environnement de travail (Thévenet, 1992; Neveu et Thévenet, 2002). Afin de comprendre l'ensemble des implications possibles, nous reprenons le concept en le définissant simplement par opposition à l'indifférence: l'engagement mesure avant tout l'intensité de la relation, quelle qu'en soit la forme (Valéau, 2001, 2004). Partant de là, de façon semblable au champ entrepreneurial, on observe un clivage entre une approche dite «comportementale» et une version «attitudinale».

L'engagement «comportemental» peut être défini comme ce qui pousse l'individu à persévérer dans une «ligne d'action» donnée. Cet attachement trouve, suivant les auteurs, différentes explications possibles:

- les investissements (side bets; Becker, 1960) effectués par l'individu dans le passé. La poursuite du comportement constitue un moyen de récupérer les coûts ainsi engagés;

- la dissonance cognitive (Festinger, 1957). La cohérence de la ligne d'action entreprise constitue, dans cette perspective, une fin en soi. Staw (1976) montre comment cette volonté de rester cohérent peut, dans certains cas, devenir de l'entêtement et mener à des surenchères irrationnelles;

3. Les Français parlent d'«implication», les Québécois d'«engagement», les anglophones de «commitment». 
- les pressions sociales s'exerçant, sur l'individu, dans le sens d'une perpétuation des comportements que l'individu a,jusque-là, donnés à voir (Pfeffer et Salancick, 1978; Beauvois et Joule, 1987). Le comportement devient alors fondamentalement social.

L'engagement «attitudinal» se réfère, pour sa part, à la façon dont l'individu perçoit et donne sens à son environnement. Suivant cette perspective, il constitue une représentation de leur relation. Ces positions seraient susceptibles d'orienter ses comportements. Suivant cette perspective, il existe tout d'abord différents modes d'implication (Etzioni, 1961; O'Reilly et Chatman, 1986; Becker, 1992; Morrow, 1993). La typologie la plus systématiquement citée est celle de Allen et Meyer (1997), elle distingue:

- l'engagement affectif fondé sur l'adhésion aux buts et aux valeurs de l'entreprise (Kelman, 1958; Etzioni, 1961; Mowday, Steers et Porter, 1979);

- l'implication normative reposant sur l'obligation morale et le devoir (Kelman, 1958; Kanter, 1968; Wiener, 1982);

- l'implication calculée qui institue l'échange d'intérêts différents (Etzioni,1961; Herbiniak et Alutto, 1972; Allen et Meyer, 1997). Ces calculs engagent l'individu (Becker, 1960; Staw, 1976). Allen et Meyer parlent d'implication «continue».

À cette typologie des modes d'engagement, on pourrait ajouter l'implication aliénante d'Etzioni (1961): certains individus souffrent de relations vécues, pour une raison ou pour une autre, comme négatives.

Ces modes d'engagement peuvent être adressés à différents objets (Reichers, 1985; Morrow, 1993; Meyer et Herscovitch, 2001). Certains sont directement liés à l'entreprise, comme, par exemple, le dirigeant, le supérieur hiérarchique ou le travail; d'autres concernent différents aspects de l'environnement de travail comme, par exemple, le client, la carrière, les collègues ou le métier.

Un certain nombre d'auteurs évoquent, par ailleurs, la possibilité d'engagements multiples. Allen et Meyer (1990) proposent d'additionner les différentes modes d'engagement liés à un objet donné comme, par exemple, celui de l'implication duale entre l'entreprise et le syndicat (Angle et Perry, 1986; Neveu, 1996). Cette multiplicité semble souvent engendrer la contradiction (Valéau,1998,2004). Nous questionnons ici les multiples engagements de l'entrepreneur: son engagement dans l'entreprise, mais également les autres engagements possibles. 


\subsection{Les points de vue de la littérature en entrepreneuriat sur l'engagement}

La littérature sur ce que «fait» l'entrepreneur peut être rapprochée de l'engagement «comportemental»; elle évoque une ligne d'action orientée vers la croissance. Une fois son entreprise créée, l'entrepreneur persévérerait dans ce sens, compte tenu de ses investissements passés. Il serait tenu d'aller toujours plus vite, toujours plus loin, de surenchérir (Staw, 1976), jusqu'à ce que l'entreprise puisse fonctionner de façon autonome.

La littérature sur ce que «pense» l'entrepreneur peut être, pour sa part, interprétée en termes d'engagement «attitudinal». La typologie de Filion (2000b), par exemple, présente un «bûcheron» centré sur son travail, un «missionnaire» mû par la réalisation de ses valeurs, un séducteur orienté sur les relations sociales, un «joueur» plus calculateur, un «converti» centré sur son produit et ses stratégies et un «vacancier» se partageant entre son entreprise et d'autres activités. Ces différentes démarches entrepreneuriales correspondent, à l'évidence, à différents modes et différents objets d'engagement. Dans ce sens, Naffziger, Hornsby et Kurato $(1994,1997)$ proposent différents points de vue: selon eux, les entrepreneurs se lancent dans la création d'entreprise avec un ensemble de buts significatifs pour eux, personnellement. Ils ajoutent que les entrepreneurs définissent leurs attentes en des termes plus larges que les mesures traditionnelles de la performance.

De plus en plus d'auteurs intègrent également des éléments liés à la vie personnelle de l'entrepreneur (Dyer, 1994; Bruyat, 1994), des arbitrages avec la famille (Landsberg, 1983; Westhead,1997) et la prise en compte de logiques communautaires (Valéau, 2001). La notion «d'entrepreneuriat social» fait aussi son chemin (Valéau, Cimper et Filion, 2005). On accorde désormais aux entrepreneurs des démarches «non seulement lucratives» (Valéau, 2001) et des engagements ne se limitant plus uniquement à l'entreprise.

Quelques contributions évoquent la genèse de cet engagement. Pour Bruyat (1994), le projet d'entreprendre constitue un projet de vie en rupture avec le salariat. Dans ce sens, Moreau (2004) insiste sur la dimension identitaire de l'entrepreneuriat: sur le fait que l'individu se définit lui-même comme entrepreneur. Cette représentation mentale lui donne une nouvelle cohérence. Selon Moreau, cette identité se construit durant la phase qui précède le passage à l'acte. Par la suite, l'entrepreneur cherchera à être reconnu et légitimé, par les autres, dans ce rôle.

Certains textes mentionnent les changements d'engagements. Selon Naffziger, Hornsby et Kurato $(1994,1997)$, les attentes des entrepreneurs changent avec le temps, avec les nouvelles opportunités, avec la réalité des 
opérations ou l'émergence de la compétition. La notion de temps et de changement est présente dans la plupart des textes sur l'entrepreneuriat avec le processus de création et de développement d'entreprise; il s'agit là de changements touchant plus directement l'entrepreneur compte tenu des événements de sa vie professionnelle, mais aussi de sa vie personnelle (Gartner et al., 1994; Kisfalvi, 2002) et des rôles qu'il endosse (Dyer, 1994). Pour ces auteurs, les engagements à poursuivre l'entrepreneuriat ne sont pas forcément les mêmes que ceux qui motivent sa création. Suivant une perspective plus détaillée, Kisfalvi (2002) montre que, suivant les entrepreneurs, certaines priorités restent relativement constantes, alors que d'autres changent.

Différents auteurs décrivent les stress liés à l'engagement entrepreneurial. Pour Boyd et Gumpert (1983), le surmenage est inévitable; il constitue un corollaire de cette expérience. Pour Adebowale (1994), le temps passé dans l'entreprise et l'immersion dans l'activité expliquent en partie ce stress, mais il accuse également un besoin d'accomplissement et des ambitions démesurées qui amènent parfois l'entrepreneur à vouloir en faire trop, à vouloir fixer la barre trop haut. Afzalur (1996) évoque des symptômes tels que l'anxiété, la dépression et l'irritabilité. Pour Adebowale (1994) et Afzalur (1996), les entrepreneurs peinent à gérer ce stress à cause du manque de soutien; Adebowale (1994) invite les entrepreneurs à en prendre conscience.

Certains abordent plus directement les difficultés, voire les échecs des entrepreneurs. Pour Shaver (1995), les vrais entrepreneurs sont ceux qui persistent à travers tous les défis, alors que les non-entrepreneurs seraient des gens qui auraient essayé une fois, qui auraient échoué et jeté l'éponge. Ces hypothèses se trouvent largement vérifiées par les résultats des recherches menées par Cardon et McGrath (1999); dans leur article «Quand les choses se gâtent... pour une psychologie de l'échec et de la remotivation des entrepreneurs ${ }^{4} »$, ils constatent que les individus attribuant leurs difficultés à la quantité d'efforts fournis se remotivent plus facilement que ceux remettant en cause leurs circonstances. Face aux contre-performances, ils redoublent d'efforts. Ces réflexions rejoignent celles de Simon, Houghton et Aquino (2000) pour qui l'impression de contrôler les choses qui caractérise les entrepreneurs favoriserait la persévérance face aux incertitudes relatives à la survie de l'entreprise et face aux coups durs. Ces éléments viennent nuancer le mythe entrepreneurial (Shaver, 1995): l'entrepreneur connaît des coups durs, mais se relève très vite. Cela dit, ces contributions restent encore sur les motivations: ces difficultés ne faisant que les renforcer. «Les entrepreneurs

4. Traduction du titre de leur article: «When the going gets tough... toward a psychological of entrepreneurial failure and remotivation». 
sont fascinants. Ils alternent entre l'extase du succès et les affres de l'échec, ils rebondissent pour revivre à nouveau. Ils ont un fort besoin d'excitation et de risques» (Afzalur, 1996, p. 1, traduction libre).

Certaines de ces contributions utilisent le mot «engagement» mais ne le définissent pas en tant que concept. La plupart parlent de motivations, d'attentes ou de satisfactions, notions qui peuvent facilement être interprétées en termes d'engagement. Ce concept, tel qu'il a été défini en GRH, nous semble pouvoir intégrer ces différentes préoccupations: il conceptualise de façon cohérente la relation entrepreneur-entreprise, conformément aux orientations ontologiques notamment introduites par Bruyat (1994) et Verstraete (2002). Il permet de réintroduire l'entrepreneur dans l'équation et de répondre aux programmes de Gartner et al. (1994): étudier ce que les entrepreneurs pensent et comment ils en arrivent à penser ce qu'ils pensent, pour comprendre ce qui se passe au cours de l'activité entrepreneuriale. Pour ces auteurs, les théories devraient refléter davantage ces expériences. Elles devraient rendre compte de ce que c'est que d'être entrepreneur du point de vue des intéressés eux-mêmes (Dyer, 1994). Il manque encore dans la littérature des contributions plus introspectives. Cette recherche s'inscrit dans cette perspective: comprendre l'engagement de l'entrepreneur en lien avec son expérience vécue. Cette expérience s'inscrit dans la durée, et cette durée implique généralement des contingences et des changements, des réussites et des difficultés, des «hauts» et des «bas». Nous explorons le processus de développement de cet engagement. Comment naît-il? Comment évoluet-il? Dans quelle mesure peut-il baisser? Dans quelle mesure l'envie d'être entrepreneur peut-elle être remise en question?

\section{Méthodes}

Nos données montrent que le doute apparaît dans presque tous les parcours d'entrepreneurs. Pourtant, le mot «doute» ne constitue clairement pas un mot clé de la littérature sur l'entrepreneuriat. Ce phénomène n'a pas encore véritablement fait l'objet de recherches scientifiques; il n'est pas ou peu évoqué par les textes, ceux-ci insistant plutôt sur la persévérance des entrepreneurs face aux difficultés. Suivant une démarche empirico-formelle (Glaser et Strauss, 1967), les «propositions » présentées dans le cadre de cet article sont essentiellement issues des réalités du terrain et constituent une formalisation théorique a posteriori capable de rendre compte de l'ensemble des données recueillies. Cette exploration qualitative nous permet d'emblée d'approfondir les «processus complexes et évolutifs » étudiés (Kisfalvi, 2002), de comprendre ces états d'âme, pour, dans un second temps, les restituer au sein de la littérature existante. 
Les phénomènes étudiés étant essentiellement de l'ordre de l'expérience vécue, nos méthodes privilégient tout naturellement l'entretien semidirectif. Nous avons travaillé sur des récits de vie. «L'unité d'analyse » (Yin, 1984) retenue pour cette recherche n'est pas l'entreprise, mais l'individu: son parcours et ses relations avec son entreprise. Nous comparons les expériences et les réactions des uns et des autres et, ce faisant, analysons en profondeur les variables individuelles. La confrontation des expériences et des réactions de nos 50 cas nous permet de modéliser un processus d'engagement entrepreneurial en quatre phases.

\subsection{Les entretiens avec les entrepreneurs}

Nous avons retenu, pour cette recherche, 50 entretiens sur un échantillon de 97 comprenant un grand nombre de petites structures. Sans vouloir prendre part au débat sur ce qu'est un entrepreneur (Gartner et al., 1994) ${ }^{5}$, nous avons préféré écarter un certain nombre de propriétaires-dirigeants n'innovant plus du tout. Nous avons gardé ceux continuant d'apprendre, de saisir des occasions d'affaires, de prendre des décisions plus ou moins risquées, impulsant encore leur entreprise. Partant de là, à des fins de comparaison, nous avons constitué un échantillon raisonné en termes de diversité (Morse, 1994), comprenant des individus issus de différentes cultures, ayant créé des entreprises petites ou grandes, œuvrant dans différents secteurs d'activité (tableau 1).

\section{TABleau 1}

\section{Échantillon}

\begin{tabular}{lcccc}
\hline Pays/région & Nombre & Taille & Activités & Ancienneté \\
\hline Région Centre (France) & 12 & & & \\
La Réunion (France) & 12 & & Agriculture & \\
Gloucestershire (Angleterre) & 12 & 5 à 100 & Industrie & $>2$ ans \\
Îles de la zone océan Indien & 7 & & Services & \\
Québec* & 7 & & & \\
\hline
\end{tabular}

* Entretiens réalisés et retranscrits par la Chaire entrepreneuriale du professeur L.J. Filion.

Nous rencontrions les entrepreneurs dans leur entreprise. Nos échanges avec eux commençaient ou se terminaient presque toujours par une visite de cette dernière. Même si l'entreprise n'était pas l'objet de notre étude, cette observation constituait un ancrage important: il permettait un minimum d'expérience partagée des réalités en question. Ces observations servaient à la fois à la réalisation des entretiens et à leur interprétation.

5. Cf. la section 1.2 .

Revue internationale P.M.E., vol. 20, nº 1, 2007 
Les entretiens proprement dits commençaient toujours par une présentation de l'entreprise: outre les informations ainsi obtenues, c'était une façon de lier connaissance sur un terrain relativement concret et objectif. Puis, nous posions à notre interlocuteur une seule et unique question: «Pourrions-nous reprendre votre parcours, en revenant à la première fois où vous avez songé à créer votre propre entreprise ? » Partant de là, nous suivions notre interlocuteur en lui demandant d'approfondir certaines idées ou en l'interrogeant sur ce qu'il ressentait et voulait à l'époque. Les entretiens partaient ainsi d'éléments relativement techniques pour prendre peu à peu une tournure très personnelle.

La partie la plus délicate de l'entretien était celle concernant les doutes. Comme le montre le tableau 4 (partie 3.2), les entrepreneurs ressentent, d'ordinaire, une certaine pudeur et, ce faisant, une certaine réticence à évoquer ce qui peut être perçu comme des moments de faiblesse. Les entretiens semi-directifs facilitent la congruence et l'acceptation inconditionnelle de soi (Rogers, 1961). Dans ce contexte «compréhensif», la plupart de nos interlocuteurs ont évoqué spontanément ces aspects de leur parcours. Dans quelques cas seulement, lorsque notre interlocuteur n'en parlait pas, nous avons demandé: «Avez- vous déjà été tenté d'abandonner? » Les témoignages ainsi obtenus étaient d'autant plus détaillés, d'autant plus approfondis et d'autant plus justes que ces ressentis étaient, bien souvent, exprimés pour la première fois.

On pourra bien évidemment reprocher à cette méthode les risques que l'interviewé ne reconstruise a posteriori les événements. Nous nous intéressons ici à l'expérience vécue; il ne s'agit pas des événements en eux-mêmes, mais de ce que l'individu a pu vivre et ressentir dans ce cadre. La conscience de l'expérience vécue, telle que nous la concevons, passe forcément par une construction de sens qu'elle soit actuelle ou rétrospective. Lorsque nos interlocuteurs étaient d'ores et déjà dans la phase de questionnement, le sens produit pouvait être tout aussi bien biaisé par une désirabilité pour soi (Paulhus, 1984), un refus ou des difficultés à accepter la situation présente. Paradoxalement, le sens produit après coup peut se révéler au moins aussi juste dans la mesure où, avec le recul, l'individu est mieux en mesure d'admettre certaines réalités. Nous pensons également que ces biais peuvent être limités par l'attitude de l'interviewer, l'acceptation inconditionnelle de l'interviewé peut l'aider à manifester plus d'authenticité (Rogers, 1961). Chaque entretien contribue à cette production de sens:le fait d'énoncer l'expérience peut ainsi amener des prises de conscience, il peut stimuler la réflexion et l'élaboration de la pensée sans pour autant modifier les ressentis initiaux. 


\subsection{L'analyse des entretiens}

Nos interprétations des discours ainsi recueillis ne prétendent pas à la « vérité» au sens «positiviste du terme», mais à une «vraisemblance» (Guba et Lincoln, 1994; Adler et Adler, 1994). Nos interprétations portent sur deux niveaux:

- au premier degré, nous retenons le sens visé par l'acteur;

- dans une seconde lecture, nous observons les discours de façon plus critique, nous les regardons comme une activité cognitive visant à donner un sens à l'expérience vécue.

Cette double lecture nous permet une analyse approfondie de chaque entretien. Elle nous facilite également, dans un troisième temps, la comparaison et la modélisation d'expériences entrepreneuriales plus ou moins avancées du point de vue des ressentis et de leur prise de conscience.

Nos interprétations s'exerçaient tout d'abord au premier degré:à partir des retranscriptions exhaustives des entretiens, nous tentions de comprendre ce que nos interlocuteurs avaient voulu signifier. Par exemple, par «J'étais fier de mon entreprise », nous comprenions un mélange d'implication affective et normative dans l'entreprise; par «On ne peut pas douter», nous comprenions simplement une absence de doute.

Puis, dans un deuxième temps, nous abordions ces mêmes discours de façon plus critique: nous analysions l'activité cognitive visant à donner un sens à l'expérience vécue, à travers différentes approches de la communication et, notamment, des éléments de linguistique (Glady et Valéau, 1996). Dans l'expression «on ne peut pas douter», nous relevions une négation, mais aussi une évocation du doute. Nous interprétions cette phrase comme une tentation refoulée: une implication ambivalente entre les ressentis et les normes ${ }^{6}$. Nous pouvions ainsi nuancer nos premières interprétations.

Dans un troisième temps, nous approfondissions l'analyse en comparant les différents entretiens. Nous avons ainsi relevé, au-delà des différents modes et objets d'engagement, d'importantes similarités: notamment, les changements et les doutes. Afin d'illustrer les changements entre les contenus de l'engagement de départ et l'engagement finalement adopté, nous avons retenu trois profils, a priori, extrêmement différents. Pour rendre compte des moments de doutes, nous avons rassemblé et juxtaposé, tous azimuts, des extraits de nombreux entretiens. À l'issue de ces analyses, nous proposons un modèle relativement simple, s'apparentant à un idéal-type (Weber,1921) : un schéma opératoire permettant de comprendre et de donner un sens à la

6. Ces questions sont développées dans la section 3.2. 
réalité, un modèle abstrait construit à partir de traits caractéristiques et singuliers. Pour Weber (1921), le type idéal n'est pas un profil moyen, mais un tableau de pensée homogène, une structure logique ordonnant et enchaînant des phénomènes isolés et diffus.

La recherche qualitative est un travail d'artisanat (Wacheux, 1996) qui présente un certain nombre de limites, mais aussi d'avantages. Nos catégories et nos remarques ne s'appliquent pas nécessairement à d'autres échantillons et elles ne sont pas forcément statistiquement représentatives. Par la suite, des réplications seront nécessaires (Yin, 1984) afin d'amender et de consolider ce modèle. Cela dit, les propositions ici développées rendent compte de l'ensemble des réalités de notre échantillon (Guba et Licoln, 1994): ancrées dans les réalités de terrain (Glaser et Strauss, 1967), elles saturent les données issues de nos 50 entretiens (Morse, 1994; Adler et Adler, 1994). Outre ces éléments de «cohérence interne» (Guba et Licoln, 1994; Adler et Adler, 1994), la «vraisemblance» de ce modèle fut confirmée de deux façons:

- d'une part, en triangulant les entretiens ainsi constitués avec sept autres menés par L.J. Filion dans le cadre de la Chaire entrepreneuriale. Ces cas supplémentaires constituaient en quelque sorte une première réplication validant la pertinence de ce modèle (Yin,1984). De plus, le fait de retrouver, dans le cadre d'entretiens menés par un autre chercheur suivant d'autres problématiques, les mêmes éléments relatifs à l'engagement et au doute nous permet de vérifier que les propos que nous avons, de notre côté, recueillis n'étaient pas induits par nos préoccupations et nos façons d'aborder les entretiens;

- d'autre part, en le restituant aux intéressés. Le fait qu'ils y trouvent un reflet acceptable, voire éclairant de leur expérience constitue un indice non négligeable de pertinence (Glaser et Strauss, 1967 ; Morse, 1994; Wacheux, 1996).

Cette première version de notre modèle suffit à alimenter ou à réfuter les théories générales (Popper, 1968). Il nous permet de «converser» (Huff, 1999) simplement avec la littérature en entrepreneuriat afin de mieux comprendre certains aspects de l'expérience entrepreneuriale.

\section{Le processus d'engagement entrepreneurial}

Compte tenu des données de terrain, nous pouvons reconstituer le développement de l'engagement sous la forme d'un processus comprenant quatre étapes. Nous évoquons les changements entre l'engagement de départ et l'implication finalement développée à travers trois exemples. Entre les deux, nous approfondissons les différents aspects des phases de persévérance et de doute à travers des extraits de nos 50 entretiens. 


\subsection{L'engagement de départ}

Des auteurs, de plus en plus nombreux, mettent en avant la diversité des entrepreneurs (Gartner et al., 1994). Ils prennent notamment en compte les liens existant entre l'entreprise et des projets personnels (Naffziger, Hornsby et Kurato, 1994; Bruyat, 1994; Verstraete, 2002). Les résultats de cette recherche confirment parfaitement ces liens; nous l'illustrons à travers trois exemples correspondant à des engagements entrepreneuriaux, a priori, très différents.

\section{Proposition 1. Les entrepreneurs démarrent leur entreprise sur la base de différents engagements.}

TABLEAU 2

\section{L'engagement de départ : trois exemples}

\begin{tabular}{|c|c|}
\hline $\begin{array}{l}\mathbf{1} \\
\text { Calculé/ entreprise } \\
\text { Normatif - identification/ } \\
\text { jeu et gain } \\
\text { Affectif/style de vie - risque }\end{array}$ & $\begin{array}{l}\text { L'entrepreneur } 1 \text { et son associé vivent leurs «heures de } \\
\text { gloire». Ils sont connus de tous. Ils font des «coups pas } \\
\text { croyables». Ils ont l'audace et la "gagne». Tout leur réussit. } \\
\text { Tout le monde dit: «ces deux-là, ils vont trop vite», tout le } \\
\text { monde attendait qu'ils se «plantent». Ces médisances ne } \\
\text { faisaient que renforcer leurs motivations à réussir. Partis de } \\
\text { rien, ils ont } 1000 \text { employés. Cela dit, le processus modélisé } \\
\text { se retrouve dans la très grande majorité des cas rencontrés. } \\
\text { Ils vivent ainsi comme ils ont commencé: sur un coup de } \\
\text { tête, par défi. }\end{array}$ \\
\hline $\begin{array}{l}2 \\
\text { Affectif et normatif } \\
\text { Identification/entreprise } \\
\text { Normatif/métier du bâtiment } \\
\text { Affectif et normatif } \\
\text { Identification/statut }\end{array}$ & $\begin{array}{l}\text { L'entrepreneur } 2 \text { a commencé à travailler très jeune :à } 16 \text { ans, } \\
\text { il a quitté l'école pour commencer comme apprenti dans une } \\
\text { entreprise du bâtiment. Là, il s'est formé à tous les métiers. } \\
\text { Et puis, il en a eu assez de se «faire exploiter» : c'est lui qui } \\
\text { «bossait et c'est le patron qui ramassait». Alors, il a «pris le } \\
\text { pas » avec un ami. Ils faisaient déjà du travail au noir. Rapi- } \\
\text { dement, ils se sont rendu compte que ce n'était pas si facile. } \\
\text { En fait, ils se retrouvaient avec moins d'argent qu'avant. Ils } \\
\text { se sont disputés, l'autre est parti, lui s'est demandé ce qu'il } \\
\text { devait faire, mais il ne voulait pas redevenir un «larbin». } \\
\text { Il s'est accroché. Avec le recul, il pense que c'était surtout } \\
\text { une question d'amour-propre. Petit à petit, il a remonté la } \\
\text { pente, les contrats sont venus, il a embauché. Son activité } \\
\text { est devenue plus importante et plus lucrative. Il fait très } \\
\text { attention à ses coûts: il n'a pas fait l'école, mais il sait faire } \\
\text { des additions et des soustractions, il sait compter ce qu'il } \\
\text { dépense et ce qu'il gagne. Il sait aussi négocier ses contrats. } \\
\text { Son entreprise emploie } 300 \text { salariés. Il a enfin le sentiment } \\
\text { d'être devenu un «monsieur», quelqu'un de très respecté. Il } \\
\text { gagne beaucoup d'argent. Il est là où il a toujours voulu être, } \\
\text { il le mérite, il s'est battu. }\end{array}$ \\
\hline
\end{tabular}

Revue internationale P.M.E., vol. 20, nº 1, 2007 
3

Calculé/ entreprise

Affectif et normatif/métier musicien

Normatif/style de vie
L'entrepreneur 3 vend des instruments de musique. Il est luimême musicien. Il a réussi à vivre sa passion. Son magasin est un lieu de rencontre de la scène locale, les gens viennent, jouent ensemble. Il a tous les modèles de guitares les plus rares. Il ne vend pas beaucoup, mais il vit avec sa passion.

Ces trois exemples correspondent à des profils relativement typiques, mais non exhaustifs de la diversité de cette population. Certains, comme l'entrepreneur 1, viennent par goût du risque, pour saisir des occasions d'affaires. D'autres, comme l'entrepreneur 2, se retrouvent dans des logiques de promotion sociale qui font sens par rapport à leur milieu d'origine. Pour d'autres encore, comme l'entrepreneur 3, l'entreprise n'est qu'un moyen de réaliser d'autres objectifs très personnels. Les engagements des entrepreneurs à l'égard de leur entreprise se révèlent ainsi comme des combinaisons subtiles et uniques d'intérêts, de sentiments et de valeurs, et nos interprétations à des fins d'analyse et de comparaison restent à certains égards réductrices. Au-delà de leur diversité, ces engagements ont en commun l'identification à la performance et la réalisation de soi; nos données rejoignent en cela les énoncés établis de longue date par la littérature entrepreneuriale (Brockhaus et Horwitz, 1985). Suivant cette perspective, l'entreprise apparaît comme un objet d'engagement commun à tous.

Comme le souligne Bruyat (1994), ces engagements à créer l'entreprise sont déjà le résultat de changements par rapport à une situation antérieure n'incluant pas cette éventualité. Comme l'évoque Moreau (2004), ils ont déjà, au moment de démarrer, reconstruit une identité d'entrepreneur. Nous approfondissons à présent la phase du tout début, le moment du démarrage, lorsque l'entrepreneur passe du rêve à la réalité.

\subsection{L'engagement dans l'action}

La théorie décrit l'entrepreneur comme quelqu'un qui jamais ne doute, qui jamais ne se laisse aller. Il reste très focalisé tout en s'adaptant; il demeure, de toute façon, très confiant (Afzalur, 1996; Simon, Houghton et Aquino, 2000; Simon et al.,2002). Ces idées font partie intégrante des mythes évoqués par Shaver (1995, p. 1, traduction libre) : «Cette hypothétique personne a un incroyable désir d'accomplissement, un besoin de contrôler les événements, il est immunisé contre le risque.» Les propos d'Isachen (1996, p. 1, traduction libre) vont dans le même sens: «Les entrepreneurs s'épanouissent dans les défis durant les moments d'incertitude [...] Ils sont toujours à la recherche du prochain défi [...] Ils sont porteurs d'espoir et d'engagement [...]» Nos données montrent que, effectivement, face à l'adversité, les entrepreneurs maintiennent l'intensité et le contenu de leur engagement initial. 
Proposition 2.

Les entrepreneurs maintiennent, dans un premier temps, leur engagement de départ. Cette persévérance constitue une obligation matérielle, affective, morale et sociale.

\section{TABLEAU 3}

\section{L'engagement dans l'action et la condition d'entrepreneur (Extraits de différents entretiens rassemblés par thème)}

\begin{tabular}{|c|c|}
\hline Résumé & Illustration \\
\hline $\begin{array}{l}\text { 1. Une activité } \\
\text { risquée }\end{array}$ & $\begin{array}{l}\text { «Être chef d'entreprise, c'est prendre des risques»- } \\
\text { «De toute façon, vous êtes remis en cause tous les matins»- } \\
\text { «Moi, en cinq ans, j'ai dû arriver deux ou trois matins l'esprit } \\
\text { tranquille dans cette entreprise, donc, tous les matins vous } \\
\text { êtes remis en cause sur vos prévisions, sur votre vision des } \\
\text { choses, donc, il faut l'esprit d'initiative.» }\end{array}$ \\
\hline $\begin{array}{l}\text { 2. Des moments } \\
\text { difficiles }\end{array}$ & $\begin{array}{l}\text { «En } 30 \text { ans de vie de l'entreprise, il y a toujours des hauts } \\
\text { et des bas.» - «Il y a des moments où on se dit c'est dur, } \\
\text { comment on va finir le mois.» - «J'ai toujours vécu avec } \\
\text { [la précarité], on n'y pense pas à longueur de journée, mais } \\
\text { bon...» }\end{array}$ \\
\hline 3. L'obstination & $\begin{array}{l}\text { «Tous les deux, on est obstinés, on ferme pas les yeux, } \\
\text { on ferme pas les yeux mais on baisse la tête pour rentrer } \\
\text { dedans.»- «Il faut être un battant.»- «Il faut avoir le goût } \\
\text { de l'aventure.» }\end{array}$ \\
\hline $\begin{array}{l}\text { 4. Beaucoup de } \\
\text { travail }\end{array}$ & $\begin{array}{l}\text { «Donc, on s'est retrouvé comme ça, travailler beaucoup } \\
\text { plus que prévu: minimum sans exaérer } 11 \text { heures par jour. } \\
\text { Malheureusement, on n'a pas baissé le rythme, on est devenu } \\
\text { plus productif. Encore aujourd'hui minimum } 11 \mathrm{~h} \text { par jour, } \\
\text { le samedi, le dimanche.»- «Parce qu'on ne peut pas laisser } \\
\text { l'entreprise comme ça, il faut constamment, constamment } \\
\text { donner de l'énergie.» }\end{array}$ \\
\hline $\begin{array}{l}\text { 5. L'obligation de } \\
\text { réussir }\end{array}$ & $\begin{array}{l}\text { «Baisser les bras, non, on peut pas, il faut faire face à ces } \\
\text { périodes-là.» - «Pendant les deux premières années, c'était } \\
\text { très tendu. On était condamné à réussir!» }\end{array}$ \\
\hline $\begin{array}{l}\text { 6. Toujours donner } \\
\text { le change }\end{array}$ & $\begin{array}{l}\text { "Il faut toujours donner aux gens l'impression que vous êtes } \\
\text { le premier, il faut garder la main sinon les gens commencent } \\
\text { à douter.»- «Il faut aller de l'avant, garder la tête haute, } \\
\text { faire voir au gens que vous êtes là pour gérer l'entreprise.»- } \\
\text { «Ne pas livrer sa perte de confiance en soi ou dans les autres. } \\
\text { Se créer un costume sur lequel on ne perçoit pas de trou.» }\end{array}$ \\
\hline
\end{tabular}

Les entrepreneurs évoquent l'incertitude comme leur condition ordinaire. Ils vivent un quotidien fait d'adversité (extraits 1 et 2). Les témoignages rejoignent sur ce point les théories: les entrepreneurs sont bien des gens qui 
prennent des risques, puis les assument de façon quasi quotidienne. Face à cette adversité, ils sont fiers de persister. Ils mettent en avant leur ténacité, leur volonté de poursuivre envers et contre tous (extraits 3). Cela dit, défier cette adversité leur donne des sentiments partagés entre un certain plaisir, un peu d'orgueil et beaucoup de stress. Les entrepreneurs présentent également cette persévérance comme une nécessité, une obligation: l'activité de l'entreprise ne leur laisse guère le choix. Ils sont pris dans l'action (extraits 4 et 5); ils «naviguent» souvent «à vue». Lorsqu'ils pensent à tout ce qu'ils ont investi, ils ne se sentent pas le droit de s'arrêter ne serait-ce que pour souffler. Cette persévérance prend parfois la forme d'une fuite en avant (extrait 5). Au-delà, le doute apparaît comme un interdit social (extrait 6).

Les entretiens comprennent, très souvent, des phrases telles que: «Même si on pense que c'est négatif, il faut regarder le côté positif des choses!» Les entrepreneurs introduisent ainsi l'éventualité du doute tout en signifiant qu'ils ne veulent pas ou ne peuvent pas s'y résigner. Ils ne l'acceptent pas, ils le refoulent, mais celui-ci n'en demeure pas moins là, à l'état latent. Pris dans l'action, les entrepreneurs n'ont généralement pas le temps de se poser de questions et de méditer sur le chemin parcouru. Ils restent sur les objets et les modes de leur engagement de départ: pour paraphraser Watzlavick, Weakland et Fisch (1975), ils vivent plus ou moins ce qu'ils avaient anticipé, mais «toujours plus que» ce qu'ils avaient anticipé. Cet engagement de départ constitue un cadre de référence à l'intérieur duquel ils poursuivent leurs efforts.

Différents auteurs, comme Boyd et Gumpert (1983), Adebowale (1994) et Afzalur (1996), évoquent les stress des entrepreneurs. Ces stress restent, à ce stade, essentiellement liés au surmenage et au sentiment de perte de contrôle; ils constituent des réactions physiques et psychiques qui, jusqu'à un certain point, contribuent à leur motivation. Cette phase d'engagement dans l'action dure plus ou moins longtemps suivant les individus, mais elle aboutit, pour la plupart d'entre eux, à un stress d'une autre nature : le doute et la remise en question.

\subsection{L'engagement en question}

Comme nous l'avons déjà mentionné, le mot «doute» ne constitue pas un mot clé de la littérature sur l'entrepreneuriat. Nous trouvons ce sentiment exprimé de façon plus directe dans le texte «rechargez vos batteries entrepreneuriales» (Stevens, 1991, p. 1, traduction libre): «Vous avez envie de laisser tomber. Vous êtes prêt à vous débarrasser de ce poids que vous appelez votre entreprise [...] Malheureusement, si vous êtes comme beaucoup d'entrepreneurs, ce qui a commencé comme une aventure n'est maintenant à 
peine plus qu'un travail. Avec les années, l'excitation et le défi de conduire sa propre entreprise ont laissé la place à la routine et à l'ennui.» Nos résultats montrent effectivement des baisses d'engagement de l'entrepreneur qui, peu à peu, perd l'envie et le plaisir de se comporter de façon entrepreneuriale et qui, ce faisant, hésite entre poursuivre et abandonner.

\section{Proposition 3.}

La plupart des entrepreneurs passent par une phase de doute plus aiguë durant laquelle leur engagement se trouve profondément remis en question.

\section{TABLEAU 4 \\ Les doutes et l'engagement en question (Extraits de différents entretiens rassemblés par thème)}

\begin{tabular}{|c|c|}
\hline Résumé & Illustration \\
\hline $\begin{array}{l}\text { 1. Des remises } \\
\text { en question } \\
\text { pratiques }\end{array}$ & $\begin{array}{l}\text { «En fait, quand on est chef d'entreprise, il faut se remettre en } \\
\text { question tous les matins, parce que rien n'est jamais acquis,, } \\
\text { donc en fait, il faut limiter les problèmes. »- «Après, j'ai baissé } \\
\text { [l'effectif], parce que ça me prenait trop de temps. J'étais un } \\
\text { peu tout seul, j'arrivais pas à encadrer. Je ne surveillais pas } \\
\text { assez mes chantiers.»- «Je pense que, de temps en temps, il } \\
\text { faut savoir se remettre en question. J'ai pris un comptable.» }\end{array}$ \\
\hline $\begin{array}{l}\text { 2. Lassitude, } \\
\text { décrochage }\end{array}$ & $\begin{array}{l}\text { «Une seconde fois, ç'a été moi qui ai pensé à arrêter, par } \\
\text { lassitude des choses. Oui!»- - Je me suis assise et me suis mise } \\
\text { à rire.»- «Par moments, tu décroches un peu parce que tu ne } \\
\text { crois plus, tu en as marre, parce que le lendemain est la même } \\
\text { chose.»- - On sait très bien que le coup de } 4 \mathrm{~h} \text { du matin est } \\
\text { épouvantable pour l'entrepreneur. Si on pouvait m'assurer de } \\
\text { dormir bien tous les matins à } 4 \mathrm{~h} \text {, je serais ravie! C'est ça le } \\
\text { problème: à ce moment-là, tu ne vivras pas bien, tu ne vois pas } \\
\text { venir les problèmes; ils ne sont pas les bons. Et le lendemain, il } \\
\text { fait jour.» }\end{array}$ \\
\hline
\end{tabular}




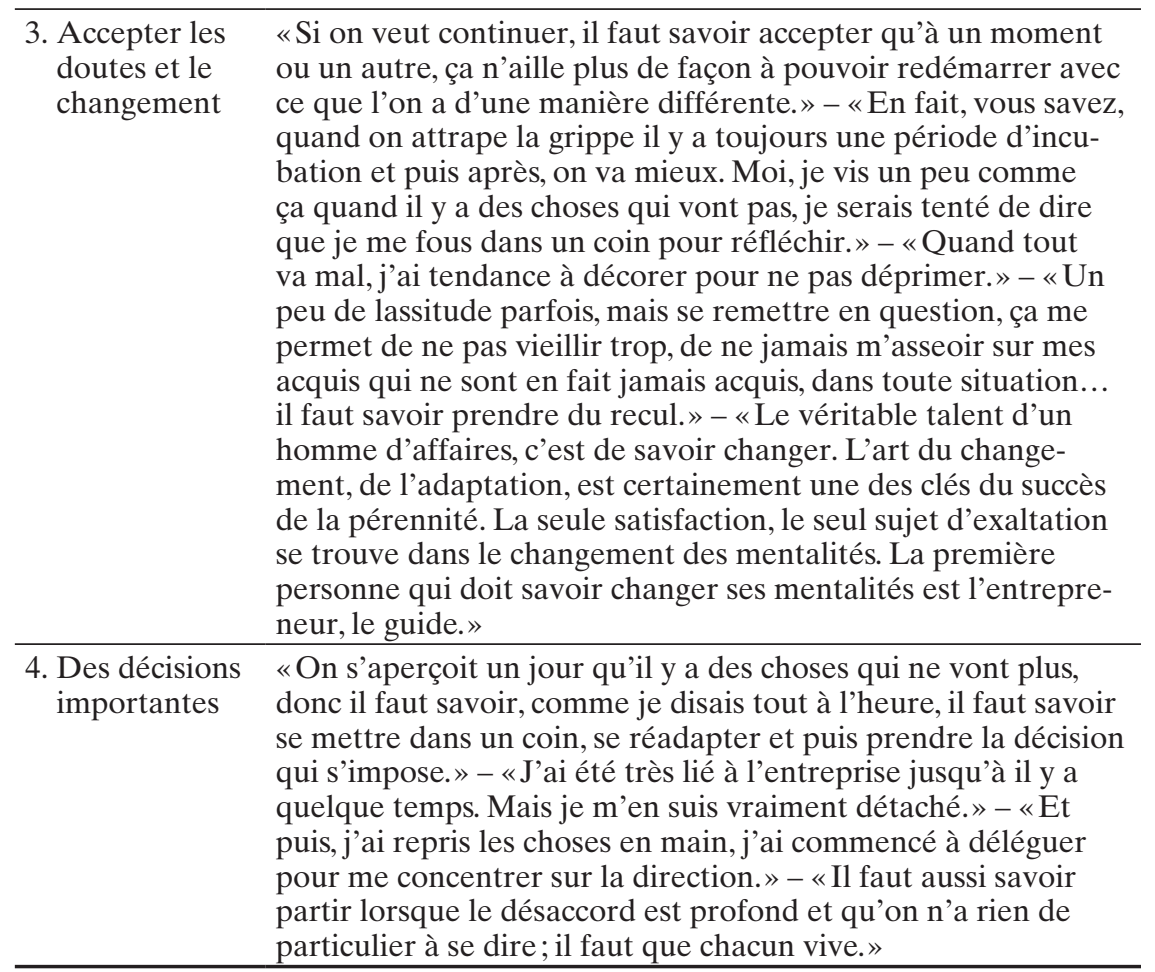

Comme dans la première série d'extraits, les entrepreneurs interviewés aiment à dire qu'ils «se remettent en question». En fait, les changements ainsi évoqués restent souvent d'ordre pratique et ne concernent généralement que l'entreprise. Ils font ainsi référence à des décisions relatives à la stratégie, au fonctionnement de l'organisation et à leur façon de travailler. Dans ce sens, les propos des entrepreneurs rejoignent la littérature:ils innovent régulièrement, dans les grandes mais aussi dans les petites choses; ils sont capables de changer leurs habitudes pour emmener l'entreprise là où ils veulent.

Les remises en question de la deuxième série d'extraits sont, en revanche, d'un tout autre ordre. Dans ces extraits, les entrepreneurs évoquent de vrais moments de doute: ceux au cours desquels ils ne savent plus s'ils veulent encore continuer. Ces doutes sont rarement exprimés d'emblée, en début d'entretien, les entrepreneurs préfèrent, au moins dans un premier temps, parler de leurs succès passés et à venir. Cette réserve s'explique également par le fait que ces ressentis ne sont pas toujours complètement analysés :ils restent souvent à l'état de sentiments flous, de contradictions et de refoulement. L'entretien accompagne parfois le passage de l'engagement dans l'action 
au questionnement. Avec la confiance et la neutralité que peuvent offrir les techniques rogériennes, les entrepreneurs peuvent parler de leurs doutes, voire, dans certains cas, en prendre conscience pour la première fois.

Ces périodes de questionnement amènent les entrepreneurs à réfléchir de nouveau sur eux-mêmes, à retracer le chemin parcouru. Suivant cette introspection, ils regardent leurs erreurs, prennent conscience de leurs émotions et de leurs sentiments, se «laissent aller» à «s'écouter» (extrait 3). Après un moment de submersion et de «lâcher prise», les entrepreneurs remettent en ordre et en sens les évènements et les émotions de leur passé. Les périodes de doutes débouchent souvent sur des décisions majeures (extrait 4), comme, par exemple, une séparation avec un associé ou l'abandon de certains marchés. Ces décisions découlent presque toujours de l'actualisation des liens entre le projet personnel et le projet d'entreprise (Bruyat, 1994): par exemple, l'entrepreneur réalisant qu'il est trop pris dans son activité peut décider de moins s'engager ou de s'engager autrement. Ces décisions marquent généralement pour l'entreprise le début d'une ère fondée sur un nouvel engagement de l'entrepreneur: sur un second souffle mieux ajusté.

\subsection{L'engagement réajusté}

Une fois le doute admis, les entrepreneurs vivent de profonds «états d'âme », ils vont «au fond des choses», puis, effectivement, ils repartent. La littérature sur l'entrepreneuriat fait très souvent état de cette capacité à repartir, à «rebondir» (p. ex., Shaver, 1995; Isachen, 1996; Cardon et McGrath, 1999). Les résultats de cette recherche montrent que cette capacité à poursuivre envers et contre tous n'est pas seulement une question de persévérance, elle peut être aussi le fruit de transformations et de reconstructions en profondeur. Les entrepreneurs font souvent preuve d'une grande capacité à changer. 


\section{Proposition 4. \\ Les engagements à poursuivre l'activité entrepreneuriale sont souvent très différents de l'engagement initial.}

\section{TABleau 5}

\section{Changement et engagement réajusté}

\begin{tabular}{l} 
Résumé \\
\hline 1. Doutes \\
Pertes de motivation
\end{tabular}
Illustration

Mais «au bout d'un moment», Louis ne savait plus quoi faire et puis ça prenait tout son temps, tout son esprit, mais il ne trouvait plus de plaisir. Il perdait l'intérêt, la motivation, il perdait «la gagne». Il a longtemps hésité. Sans doute aurait-il pu continuer longtemps comme ça. Sur le secteur, il y avait un jeu de concentration. La concurrence était très vive. Il fallait de plus en plus d'activité pour rester rentable. Il fallait «manger» les autres pour ne pas être «mangé». Louis n'a finalement pas souhaité relever ce nouveau défi, il a préféré

Vente

Petite affaire vendre ses parts à son associé. Aujourd'hui, il a une petite affaire d'une quinzaine de personnes qu'il contrôle parfaitement. Il admet être un peu «en sous-régime », mais il n'en est pas moins leader sur son marché: la location de voitures. C'est un art

Équilibre que de maintenir l'équilibre: cet équilibre entre sa vie privée et son entreprise. De fait, il pense qu'il utilise encore toutes ses compétences pour

Recul rester efficace en un temps de travail raisonnable. Il dit avoir la sagesse, avoir beaucoup plus de recul qu'à 20 ans.

2. Altercation

Et puis, un jour, il y a eu cette altercation, il se souvient qu'un de ses employés lui a dit: «Vous dites que vous êtes comme nous parce que vous avez commencé à la base, mais vous êtes comme les autres patrons, tout ce qui vous intéresse, c'est

Prise de conscience l'argent.» Ce jour-là, il dit avoir pris une «claque». Il avait «honte». Alors, il a décidé de changer. Aujourd'hui, tout en gardant la rentabilité raison-

Ouverture au bien-être de ses salariés nable, il est «heureux» et «fier » d'investir dans la sécurité et le bien-être de ses employés. Il fut ainsi Réconciliation avec ses origines l'un des premiers de sa ville à passer aux 35 heures hebdomadaires. 
3. Mariage, paternité

Besoins

Dernière chance

Professionnalisation
Et puis, il s'est marié, il a eu son premier enfant. Cette vie de bohème, «ça ne pouvait plus durer». Le magasin ne rapportait pas assez... en tout cas, pas assez pour qu'ils puissent en vivre tous les trois. Il s'était résolu à vendre, il voulait retrouver une situation plus «convenable» et plus sûre. En même temps, il ne pouvait pas s'imaginer à l'usine, avoir un patron, «tout ça». Alors, au dernier moment, il s'est dit qu'il voulait essayer une dernière fois. Il s'est donné six mois. À partir de cet instant, il a tout changé dans sa façon de faire. Il a maintenu une gamme variée mais beaucoup plus réduite, il s'apercevait en effet que, quand les gens ont trop de choix, ils n'arrivent plus à choisir. Et puis, il a commencé à s'intéresser à tous les instruments, toujours un choix de produits de qualité sur le haut de gamme. Il a réduit ses stocks. Le bouche à oreille opère, les gens venaient de plus en plus pour acheter. Il a refait son enseigne, il a pris plus de son temps pour communiquer. Il a troqué son image de musicien pour celle d'un professionnel de la musique. Maintenant, il peut dire qu'il gagne bien sa vie. Il est devenu plus entreprenant «sur le tard».

Les périodes de doute constituent des points de passage soit vers un abandon, soit vers un nouvel engagement mieux adapté aux réalités rencontrées. Comme dans l'exemple 1 (tableau 5), certains entrepreneurs limitent et focalisent davantage leur engagement: suivant un principe d'efficience, ils recherchent un rendement maximisé sous contrainte d'un temps délibérément plus limité. Inversement, comme dans l'exemple 3 (tableau 5), certains entrepreneurs, après avoir un temps privilégié le plaisir et l'inspiration, trouvent un nouveau souffle économiquement plus offensif. Au-delà de l'intensité, ces ajustements touchent le contenu même de l'engagement. Comme dans l'exemple 2 (tableau 5), les entrepreneurs évoluent souvent d'un engagement relativement simple vers un engagement plus complexe intégrant des objets plus nombreux et combinant des modes plus subtils. Dans cet exemple, l'entrepreneur intègre une responsabilité sociale qui, confrontée à l'engagement dans l'entreprise, peut aboutir à des dilemmes l'obligeant à des arbitrages plus difficiles (Valéau, 2007). Au-delà des contenus, après les doutes, c'est la nature même de l'engagement qui se trouve modifiée: elle ne découle plus de rêves et d' «a priori»; elle est ancrée dans la réalité, les ressentis et les motivations actuels; elle est plus «congruente» au sens de Rogers (1961). 


\title{
4. Un modèle du processus d'engagement entrepreneurial
}

Les trois parcours entrepreneuriaux présentés dans les tableaux 2 et 5 ont en commun le changement d'engagement. Ils donnent à voir des modifications dans les stratégies de l'entreprise, mais aussi et surtout des évolutions des individus dans leur façon d'être entrepreneurs, dans leur manière de voir et de faire l'entreprise. Les données introduites dans les tableaux 3 et 4 permettent de mieux analyser les périodes de transition, de mieux comprendre comment elles s'opèrent. Nous les rassemblons à présent dans le cadre d'un même modèle. La figure 1 décrit le processus suivant lequel les individus désirant devenir entrepreneurs se confrontent à la réalité. Suivant cette perspective, l'engagement constitue une forme d'interface, à la fois cognitive et conative, entre eux et cette dernière : il correspond à la façon dont ils se représentent leur relation avec elle, leurs attitudes à son égard. Ainsi, cette recherche montre que si, comme l'indique Moreau (2004), l'identité entrepreneuriale commence avant le passage à l'acte, son développement passe ensuite par un processus relativement long et compliqué.

\section{FiguRE 1}

\section{Le processus d'engagement entrepreneurial}

\author{
Engagement de départ \\ Engagement dans \\ l'action \\ Doutes et confusion \\ Engagement \\ en question
}

Entrepreneur Réalité

Engagement ajusté

Sens

Représentation Perceptions Attitude

Les quatre rectangles centraux de la figure 1 reprennent les quatre stades d'évolution de l'engagement tels que nous les avons mis au jour dans le cadre de l'analyse. Les flèches latérales situent les sources de l'engage- 
ment entre motivations (intérêts, valeurs et sentiments) et perceptions de la réalité. Le sens de cette confrontation évolue au gré des étapes du processus entrepreneurial:

- Dans le cadre de la première étape, l'engagement de départ reflète la volonté de l'entrepreneur, qui projette de transformer la réalité à l'image de sa «vision»;

- Dans le cadre de l'engagement dans l'action, une fois posées les premières impressions, avec l'engagement dans l'action, l'activité cognitive et conative semble rester en suspens. L'entrepreneur n'a plus de recul; il ne cherche plus à produire de définition générale de la situation, il est pris dans «l'ici et maintenant» et tente de «faire face»;

- Durant la phase de doute, la réalité reprend le dessus et revient s'imposer à l'entrepreneur. Celui-ci prend conscience de certaines données de la réalité, ces perceptions remettant en question tout ou partie de ses motivations et de sa volonté de départ;

- L'engagement ajusté réintroduit un relatif équilibre entre «réalisme» et «volonté »; l'entrepreneur finit par accepter certaines données et, après avoir adapté une partie de ses motivations, il continue à vouloir changer la réalité à travers l'entreprise qu'il «impulse».

Le processus d'engagement entrepreneurial est à la fois conatif et cognitif; c'est une découverte de la réalité associée à une transformation identitaire psychologique et sociale. Nous détaillons à présent les différentes étapes de ce processus.

Nous confirmons tout d'abord l'idée de Naffziger, Hornsby et Kurato (1994): les entrepreneurs entrent en affaires pour des raisons très différentes, avec des conceptions très différentes de leur entreprise. Ces engagements correspondent à des réinterprétations très personnelles des «mythes » de l'entrepreneur évoqués par Shaver (1995): en effet, les entrepreneurs ne sont pas toujours très réalistes non seulement par rapport aux objectifs technicoéconomiques de leurs entreprises (Pinfold, 2001), mais aussi par rapport au style de vie auquel ils imaginent pouvoir accéder.

Une fois entrés en affaires, les entrepreneurs suivent un processus commandé par l'action. À ce stade, l'entrepreneuriat apparaît comme une activité particulièrement «engageante» au sens «comportemental» du terme. Plus ils se comportent de façon entrepreneuriale, plus les entrepreneurs se sentent obligés de persévérer, voire de surenchérir dans ce sens, de façon parfois irraisonnable (Staw, 1976; Beauvois et Joule, 1987). Cette implication 
peut, ce faisant, devenir plus aliénante; les entrepreneurs ont parfois le sentiment d'être pris dans un engrenage: de ne plus avoir le choix (Etzioni, 1961; Penley et Gould,1988). Cette partie du processus d'engagement entrepreneurial, sa relative irréversibilité, peut être rapprochée des théories de Becker (1960), de Staw (1976) et de Beauvois et Joule (1987). Les pressions sociales ressenties (extrait 6) rejoignent les théories de Pfeffer et Salancick (1978). Les entrepreneurs expriment souvent des sentiments partagés entre plaisir et souffrance: ils trouvent l'expérience exaltante et, en même temps, ils vivent un stress qui, de positif, peut devenir négatif. Souvent, les entrepreneurs souffrent tout en «s'accrochant» de façon très résolue.

Le passage par le doute et la confusion se retrouve chez Piaget (1971), Watzlavick, Weakland et Fisch (1975) et Freud (1968). Pour Piaget (1971), puis pour Watzlavick, Weakland et Fisch (1975): le passage d'une représentation à une autre plus adaptée passe par une phase de déconstruction (Piaget, 1971) caractérisée par un moment de confusion (Watzlavick, Weakland et Fisch, 1975). Ce processus d'engagement peut être rapproché du processus de deuil (Freud, 1968). Pour Comte-Sponville (1995), il y a deuil chaque fois qu'il y a perte, refus ou frustration. Pour lui, la souffrance liée à ces renoncements est proportionnelle à l'attachement qui les précédait. Dans le cas des entrepreneurs, le deuil constitue un renoncement à tout ou partie des attentes et des rêves sur la base desquels ils avaient décidé de s'engager. Dans ce sens, le maintien de l'engagement initial qui caractérise la phase de projection dans l'action peut ainsi être rapprochée d'un «déni».

Une fois ce deuil consommé, les entrepreneurs peuvent enfin réinvestir de nouveaux objets: ils peuvent à nouveau s'engager. Ce passage par le doute rappelle également les «transitions» des modèles de changement: la «décristallisation» et l'«état transitoire» du modèle de Lewin (1952); la confusion émotionnelle des modèles de Scott et Jaffe (1992) et Perlman et Takacs (1990); le choc, la résistance et les explorations du modèle de Bareil et Savoie (2003). Pour nous, le doute des entrepreneurs est tout autant cognitif et conatif.

Si, comme le mentionnent Gartner et al. (1994), Dyer (1994), Naffziger, Hornsby et Kurato (1994), l'engagement change, nous ajoutons que le doute et la confusion constituent un préambule nécessaire à toute transformation. Pour Naffziger, Hornsby et Kurato (1994), les entrepreneurs poursuivent leurs activités dans la mesure où leurs attentes sont satisfaites. Pour eux, comme pour Gartner et al. (1994) et Dyer (1994), ces attentes changent. Nos résultats montrent que, dans un premier temps, les entrepreneurs continuent à se comporter de façon entrepreneuriale indépendamment des réalités rencontrées; ils persévèrent, cherchant à imposer leur vision à la réalité. Ce 
n'est que plus tard, durant la phase de doute, que les entrepreneurs intègrent véritablement certaines réalités de la situation. Ces prises de conscience les plongent tout d'abord dans la confusion avant qu'ils ne retrouvent enfin un équilibre, un engagement ajusté entre leurs motivations et leur environnement. Au final, certains engagements demeurent, mais d'autres changent en fonction non seulement de leur importance (Kisfalvi, 2002), mais également de leur compatibilité avec les réalités vécues.

\section{Proposition 5.}

Au-delà de la persévérance, le doute et la confusion sont nécessaires pour que l'entrepreneur adapte son engagement aux réalités rencontrées.

Le processus d'engagement dans l'activité entrepreneuriale se distingue des autres expériences, notamment salariées (Valéau, 1998, 2007) pour au moins deux raisons :

- la nature de l'activité entrepreneuriale: la création et le développement d'une entreprise exigent un temps de travail plus élevé que celui habituellement demandé aux salariés. De plus, les risques assumés de façon quasi quotidienne accaparent les entrepreneurs; ils n'ont pas le loisir de réfléchir sur eux-mêmes;

- le degré de ténacité des entrepreneurs. Ces derniers ont vocation à façonner la réalité conformément à leur vision; ils ont souvent cette capacité à maintenir leur engagement et leur ligne d'action face à l'adversité. Nous confirmons des capacités d'adaptation et d'innovation supérieures à la moyenne, mais nous nuançons : ces adaptations restent, au moins dans un premier temps (projection), dans le cadre de la même «vision» fondée sur l'implication de départ ${ }^{7}$.

Une fois engagé, tout pousse l'entrepreneur à persévérer dans la «ligne d'action» initiée (Becker, 1960; Staw, 1976; Beauvois et Joule, 1987). L'internalité plus élevée que d'ordinaire (Brockhaus et Horwitz, 1985) et les pressions sociales (Pfeffer et Salancick, 1978; Weick, 1979; Gartner, Bird et Starr, 1992) renforcent l'irréversibilité de cet engagement (Bruyat, 1994). Même si dans certains cas le doute intervient après une année d'exercice, il aura souvent tendance à être repoussé et reporté. Le processus d'engagement dans l'activité entrepreneuriale sera ainsi souvent plus intense et plus contrarié que d'ordinaire.

7. Toujours plus des mêmes cadres de référence (Watzlavick, Weakland et Fisch, 1975). 


\section{Conclusion}

Le passage par le doute peut se produire à des périodes différentes, mais il apparaît dans la plupart des biographies constituées dans le cadre de nos entretiens. Cette étape du processus entrepreneurial n'est que très rarement évoquée dans la littérature, pourtant, elle peut avoir un impact des plus importants sur les orientations, voire la survie de l'entreprise. La période de doute proprement dite survient lors de rendements plus incertains, qui, s'ils se prolongeaient indûment, pourraient menacer la santé de l'entreprise. Le second souffle peut ensuite donner lieu à des réorientations importantes: des réorientations non plus dictées par l'environnement et les évolutions du marché, mais à nouveau impulsées par l'entrepreneur.

Cette recherche ne constitue qu'une première exploration du processus d'engagement dans l'activité entrepreneuriale. Par la suite, il conviendrait de valider quantitativement les propositions introduites, d'examiner plus en profondeur les causes et les conséquences des différentes étapes et de mesurer les variations possibles autour de ce modèle général. Sur le plan qualitatif, nous pensons qu'il serait intéressant d'explorer les possibilités de voir ce processus se répéter à plusieurs reprises tout au long de la vie de l'entrepreneur, car certains entretiens montrent que des changements de situation ou des évolutions personnelles peuvent donner lieu à de nouvelles remises en question. Sur le plan théorique, ces futures recherches pourraient emprunter davantage aux cadres conceptuels de la gestion des ressources humaines, en particulier à ceux relatifs au développement vocationnel et à la gestion de carrière. En effet, même si l'entrepreneuriat est une aventure hors du commun, il n'en reste pas moins une expérience très humaine faite de joies et de peines, de hauts et de bas. Comme pour les autres métiers, le travail des entrepreneurs reste soumis aux contingences de leur engagement.

Sur le plan pratique, l'exploration de ce processus implique de nombreuses considérations en matière d'accompagnement des entrepreneurs. Suivant cet article, on naît plus ou moins entreprenant, mais il est par ailleurs possible de le devenir davantage, ce à quoi l'accompagnateur peut contribuer (Verstraete, 1997; Académie de l'entrepreneuriat, 2005). Suivant cette perspective, sa mission est, bien évidemment, d'accompagner l'entrepreneur dans l'élaboration et la mise en œuvre de son plan d'affaires. Elle consiste, selon nous, tout autant à l'aider à évoluer dans le cadre d'une expérience radicalement nouvelle, intéressante, stimulante mais néanmoins difficile. Il s'agirait ainsi, concrètement, de préparer les entrepreneurs à cette épreuve en les prévenant qu'ils passeront par des moments de découragement, que ces derniers sont normaux, que la plupart des autres entrepreneurs passent par là, puis redémarrent. Ensuite, suivant des méthodes de l'ordre du «counsel- 
ling» (Rogers, 1961; Priel, 2004), l'accompagnateur peut aider l'entrepreneur à reconstruire son engagement et son projet. Ce soutien psychologique aux entrepreneurs contribuerait, sans doute, à la réussite de leurs entreprises.

\section{Bibliographie}

ACADÉMIE DE L'ENTREPRENEURIAT (2005), IVe Congrès «L'accompagnement en situation entrepreneuriale», 24 et 25 novembre, Paris.

Adebowale, A. (1994), «Coping with entrepreneurial stress », Journal of Small Business Management, vol. 32, $\mathrm{n}^{\circ} 1$.

Adler, P.A. et P.Adler (1994), «Observational techniques», dans N.K. Denzin et Y.S. Lincoln (dir.), Handbook of Qualitative Research, Thousand Oaks, Sage.

AfZalur, R. (1996), «Stress, strain, and their moderators: an empirical comparison of entrepreneurs and managers », Journal of Small Business Management, vol. $34, \mathrm{n}^{\circ} 1$.

Allen, N.J. et J.P. Meyer (1990), «The measurement and antecedents of affective, continuance and normative commitment to the organisation», Journal of Occupational Psychology, vol. 63, p. 1-18.

Allen, N.J. et J.P. Meyer (1997), Commitment in the Workplace: Theory, Research and Application (Advanced Topics in Organizational Behavior), Thousand Oaks, Sage.

Angle, H.L. et J.L. Perry (1986), «Dual commitment and labor-management relationship climates », Academy of Management Journal, vol. 29, p. 31-50.

BAREIL, J. et D. SAVOIE (2003), «Comprendre et mieux gérer les individus en situation de changement organisationnel», dans R. Jacob et al. (dir.), Changement dans les organisations, Montréal, Gestion.

Beauvois, J.-L. et R.-V. Joule (1987), Petit traité de manipulation à l'usage des honnêtes gens, Grenoble, Presses universitaires de Grenoble.

BeCKer, H.S. (1960), «Notes on the concept of commitment», The American Journal of Sociology, vol. 66, p. 32-40.

BECKER, T.E. (1992), «Foci and bases of commitment: are they distinctions worth making?», Academy of Management Journal, vol. 35, n 1, p. 232-244.

Boyd, D.P. et D.E. Gumpert (1983), «The effects of stress on early-stage entrepreneurs», Actes de la conference annuelle «Frontiers of Entrepreneurship Research », Center for Entrepreneurial Studies, Babson College.

BrockHaus, R.H. et P.S. Horwitz (1985), «The psychology of the entrepreneur», dans D.L. Sexton et R.W. Smilor (dir.), The Art and Science of Entrepreneurship, Cambridge, Mass., Ballinger.

BRuyat, C. (1994), «Contributions épistémologiques au domaine de l'entrepreneuriat», Revue française de gestion, vol. 101, p. 87-99.

Revue internationale P.M.E., vol. 20, nº 1, 2007 
Bygrave, W.D. et C.W. Hofer (1991), «Theorizing about entrepreneurship », ETP, vol. 16, n 2, p. 13-22.

CANTILlon, R (1755), Essai sur la nature du commerce en général, Londres, Fetcher Gyler.

CARDon, G. et R. McGrath (1999), «When the going gets tough... toward psychology of entrepreneurial failure and re-motivation», Frontiers of Entrepreneurship Research, Center for Entrepreneurial Studies, Babson College.

Carland, J.W., F. Hoy, W.R. Boulton et J.C. Carland (1984), «Differentiating entrepreneurs from small Business owners: a conceptualization», Academy of Management Review, vol. 9, n 2, p. 354-359.

Comte-Sponville, A. (1995), Deuil, vivre c'est perdre, Paris, Autrement.

DAVIDSON, P. et J. WIKLUND (2001), «Level of analysis in entrepreneurship research: current research practice and suggestions for the future», ETP, vol. $25, \mathrm{n}^{\circ} 4$.

Davidson, P., M.B. Low et M. Wright (2001), «Low and McMillan ten years on», Entrepreneurship Theory and Practice, vol. 25, n 4 , p. 5-16.

DYER, W.G. (1994), «Toward a theory of entrepreneurial careers», ETP, vol. 19, $\mathrm{n}^{\circ} 2$.

Etzioni, A. (1961), A Comparative Analysis of Complex Organisations: On Power, Involvement, and Their Correlates, New York, The Free Press.

Festinger, L. (1957), A Theory of Cognitive Dissonance, Stanford, Cal., Stanford University Press.

Filion, L.J. (1997), «Le champ de l'entrepreneuriat: historique, évolution, tendances », Revue internationale PME, vol. 10, $\mathrm{n}^{\circ}$ 2, p. 129-172.

Filion, L.J. (2000a), «Typologie d'entrepreneur: est-ce vraiment utile?» dans T. Verstraete (dir.), Histoire d'entreprendre: les réalités de l'entrepreneuriat, Colombelles, EMS.

Filion, L.J. (2000b), «Six types de propriétaires-dirigeants de PME», Revue Organisations et Territoires, vol. 9, $\mathrm{n}^{\circ}$ 1, p. 5-16.

Freud, S. (1968), Métapsychologie, Paris, Gallimard.

GARTNER, W.B. (1998), «Who is an entrepreneur? Is the wrong question», American Journal of Small Business, printemps.

GARTNER, W.B., B.J. BIRD et J.A. STARR (1992), «Acting as if: differentiating entrepreneurial from organizational behaviour», Entrepreneurship Theory \& Practice, vol. $16, \mathrm{n}^{\circ} 3, \mathrm{p} .13-32$.

GartneR,W.B. et E. Gatewood (1992), «Thus the theory of description matters most», Entrepreneurship Theory and Practice, vol. 17, $\mathrm{n}^{\circ}$ 1, p. 5-10.

Gartner, W.B., K.G. Shaver et E. Gatewood (2000), «Doing it for yourself: career attributions of nascent entrepreneurs », Babson College, Kauffman Foundation Entrepreneurship Research Conference. 
Gartner, W.B., K.G. Shaver, E. Gatewood et J.A. Katz (1994), «Finding the entrepreneur in entrepreneurship», Entrepreneurship Theory et Practice, vol. 18, $\mathrm{n}^{\circ} 3$, p. 5-10.

Glady, M. et P. VAlÉAU (1996), «Le discours, expression ou construction de l'implication? », Paris, AGRH.

Glaser, B.G. et A.L. Strauss (1967), The Discovery of Grounded Theory. Strategies of Qualitative Research, New York, Aldine.

GuBA, E.G. et Y.S. LiNCOLN (1994), «Competing paradigms in qualitative research», dans N.K. Denzin et Y.S. Lincoln (dir.), Handbook of Qualitative Research, Thousand Oaks, Sage.

Herbiniak, L.G. et J.R. Alutto (1972), «Personal and role-related factors in the development of organizational commitment », Administrative Science Quarterly, vol. 17, p. 555-573.

Hofer, C.W. et W.D. Bygrave (1992), «Researching entrepreneurship», Entrepreneurship Theory and Practice, vol. 16, no 3, p. 91-100.

Huff, A.S. (1999), Writing for Scholarly Publication, Londres, Sage.

Isachen, O. (1996), Joining the Entrepreneurial Elite, Psychologists, Consulting Psychologists Press.

Johnson, B.R. (1990), «Toward a multidimensional model of entrepreneurship: the case of achievement motivation and the entrepreneur », ETP, vol. 14, n ${ }^{\circ} 3$.

KANTER, R.M. (1968), «Commitment and social organization mechanisms in utopian communities », American Sociological Review, vol. 33, p. 499-517.

Kelman,H.C. (1958), «Compliance, identification and internalization - three processes of attitude change», Journal of Conflict Resolution, vol. 2, p. 51-60.

KiRZNER, I.M. (1993), «The morality of pure profit», Journal des économistes et des études humaines, vol. 4, p. 315-328.

Kisfalvi, V. (2002), «The entrepreneur's character, life issues, and strategy making. A field study », Journal of Business Venturing, vol. 17, p. 489-418.

Knight, F.H. (1921), Risk, Uncertainty and Profit, Boston, Mass., Houghton Mifflin.

LANDSBERG, I.S. (1983), «Managing human resources in family firms: the problem of instritutional overlap », Organization Dynamics, vol. 12, n 1, p. 39-46.

Lewin, K. (1952), Field Theory in Social Science, New York, Harper.

Meyer, J.P. et L. Herscovitch (2001), «Commitment in the workplace: toward a general model», Human Resource Management Review, vol. 11, p. 299-326.

Mill, J.S. (1848), Principle of Political Economy with Some of Their Applications to Social Philosophy, Boston, Mass., Parker.

Moreau, R. (2004), «L'identité entrepreneuriale de soi», Revue internationale PME, vol. 17, n' 2, p. 3-16.

Morrow, P.C. (1993), The Theory and Measurement of Work Commitment, Greenwich, Conn., JAI Press Inc.

Revue internationale P.M.E., vol. 20, nº 1, 2007 
Morse, J.M. (1994), «Designing funded qualitative research», dans N.K. Denzin et Y.S. Lincoln (dir.), Handbook of Qualitative Research, Thousand Oaks, Sage.

Mowday, R.T., R.M. STeER et L.W. Porter (1979), «The measurement of organizational commitment», Journal of Vocational Behavior, vol. 14, $\mathrm{n}^{\circ}$ 2, p. 224-247.

NAFFZigER, D.W., J.S. Hornsby et D.F. KuRATo (1994), «A proposed research model of entrepreneurial motivation », Entrepreneurship Theory and Practice, vol. 18, $\mathrm{n}^{\circ} 3$, p. 29-42.

Neveu, J.P. (1996), La démission du cadre d'entreprise : étude sur l'intention de départ volontaire, Paris, Economica, $181 \mathrm{p}$.

Neveu, J.P. et M. Thévenet (2002), L'implication au travail, Paris, Vuibert.

O'Reilly, C.A. III et J. Chatman (1986), «Organizational commitment and psychological attachement», Journal of Applied Psychology, vol. 71, p. 492-499.

Paulhus, D. (1984), «Two-component models of socially desirable responding», Journal of Personality and Social Psychology, vol. 46, p. 598-609.

Penley, L.E. et S. Gould (1988), «Etzioni's model of organizational involvement: a perspective of understanding commitment to organizations », Journal of Organizational Behavior, vol. 9, p. 43-59.

Perlman, D. et G.J.Takacs (1990), «The 10 stages of change », Nursing Management, vol. 21, p. 33-38.

Pfeffer, J. et G.R. SAlAnCick (1978), «A social information processing approach of job attitude and task design», Administrative Science Quarterly, vol. 23, p. 224-253.

Piaget, J. (1971), «Inconscient affectif et inconscient cognitif», Raison Présente, vol. 19, Éditions Rationalistes.

Pinfold, J. (2001), «The expectations of new business founders», Journal of Small Business Management, vol. 39, $\mathrm{n}^{\circ}$ 3, p. 279-285.

Popper, K. (1968), The Logic of Scientific Discovery, $1^{\text {re }}$ édition 1934, New York, Huchingson.

Priel, J.M. (2004), «Éléments historiques à propos du counselling», AFCACP, site Web: <www.geocities.com/afcacp>.

ReICHERs, A.E. (1985), «A review and reconceptualization of organizational commitment », Academy of Management Journal, vol. 10, n 3, p. 465-476.

Rogers, C.R. (1961), On Becoming a Person, Boston, Mass., Houghton Mifflin Company.

SAY, J.B. (1803), Traité d'économie politique, Paris, Economica.

SChumpeter, J.A. (1935), Théorie de l'évolution économique, Paris, Dalloz.

Scott, C.D. et D.T. JAFFe (1992), Maîtriser les changements dans l'entreprise, Laval, ARC.

Shane, S. et S. Vankatamaran (1997), «The promise of entrepreneurship as a field of research », Academy of Management Review, vol. 25, n 1, p. 217-226. 
Shaver, K.G. (1995), «The entrepreneurial personality myth», BetE Review, avriljuin.

Simon, M., B. Elango, S.M. Houghton et S. SAvelli (2002), «The successful product pioneer: maintaining commitment while adapting to change », Journal of Small Business Management, vol. 40, n 3, p. 187-203.

Simon, M., S.M. Houghton et M. Aquino (2000), «Cognitive biases, risk perception and venture formation », Journal of Business Venturing, vol. 14, $\mathrm{n}^{\circ} 5$.

Smith, N.R. (1967), The Entrepreneur and His Firm: The Relationship between Type of Man and Type of Company, Bureau of Business Research, East Lansing, Michigan State University Press.

Staw, B.M. (1976), «Knee-deep in the big muddy: a study of escalating commitment to a chosen course of action», Organizational Behavior and Human Performance, vol. 76, p. 27-44.

Stengers, I. (1987), D'une science à l'autre. Des concepts nomades, Paris, Seuil.

Stevens, W.S. (1991), «Recharge your entrepreneurial batteries», Professional Press, US.

Thévenet, M. (1992), Impliquer les personnes dans l'entreprise, Paris, Liaisons, $205 \mathrm{p}$.

Ucbasaran, D., P. Westhead et M. Wright (2001), «The focus of entrepreneurial research: contextual and process issues », Entrepreneurship Theory \& Practice, vol. $25, \mathrm{n}^{\circ} 4$, p. $57-80$.

VALÉAU, P. (1998), La gestion des volontaires dans les associations humanitaires, Thèse, Université de Lille I, <www.valeau.com $>$.

VAlÉAU, P. (2001), «Pour une version non seulement lucrative de l'entrepreneur: exemples tirés de la zone Océan Indien », Management international, vol. 6, $\mathrm{n}^{\circ} 1$, p. 33-41.

VALÉAU, P. (2004), «Gérer l'implication dans le respect des différences », Habilitation à diriger les recherches, Université de Lille I.

VALÉAU, P. (2007), «La responsabilité sociale des entrepreneurs: des discours à la pratique », Humanisme et entreprises, à paraître.

VAlÉAU, P., P. Cimper et L.J. Filion (2005), «L'entrepreneuriat dans les organisations à but non lucratif», Cahier de recherche de la Chaire Rogers-Bombardier.

Van de Ven, A., R. Huston et D.M. Schroeder (1984), «Designing new business start-ups », Journal of Management, vol. 10, $\mathrm{n}^{\circ} 1$.

Verstraete, T. (1997), «Cartographie cognitive et accompagnement du créateur d'entreprise», Revue internationale PME, vol. 10, $\mathrm{n}^{\circ} 1$.

Verstraete, T. (2002), Essai sur la singularité de l'entrepreneuriat comme domaine de recherche, ADREG, 2-9518007-0-3.

Vesalainen, J. et T. PihKala (1999), «Motivation structure and entrepreneurial intentions», dans les Actes de la Conférence annuelle sur l'entrepreneurship «Frontiers of Entrepreneurship Research», Babson College.

Revue internationale P.M.E., vol. 20, nº 1, 2007 
Vesper, K.H. (1980), New Venture Strategies, Englewood Cliffs, Prentice Hall.

Wacheux, F. (1996), Méthodes qualitatives et recherche en gestion, Paris, Economica.

Watzlavick, P., J. Weakland et H. Fisch (1975), Changement, paradoxes et psychothérapie, Paris, Seuil, Coll. «Point».

Weber, M. (1921), Economy and Society, New York, Bedminster Press.

WeIck, K.E. (1979), The Social Psychology of Organizing, Reading, Mass., AddisonWestley.

WestheAd, P. (1997), «Ambitions, external environment and strategic factor differences between family and non-family companies», Entrepreneurship et Regional Development, vol. 9, $\mathrm{n}^{\circ}$ 2, p. 127-157.

Wiener, Y. (1982), «Commitment in organization: a normative view», Academy of Management Review, vol. 7, p. 418-428.

Yin, R.K. (1984), Case Study Research: Design and Methods, Beverly Hills, Cal., Sage. 